\title{
The Effect of Unreliable LLR Storage on the Performance of MIMO-BICM
}

\author{
Clemens Novak* ${ }^{*}$ Christoph Studer ${ }^{\ddagger}$, Andreas Burg ${ }^{\ddagger}$, and Gerald Matz* \\ * Institut für Nachrichtentechnik und Hochfrequenztechnik, Vienna University of Technology \\ Gusshausstrasse 25/389, 1040 Vienna, Austria; e-mail: \{clemens.novak,gmatz\}@nt.tuwien.ac.at \\ $\ddagger$ Department of Information Technology and Electrical Engineering \\ ETH Zürich, 8092 Zürich, Switzerland; e-mail: studerc@ nari.ee.ethz.ch; apburg@iis.ee.ethz.ch
}

\begin{abstract}
Future semiconductor technologies will suffer from reliability issues, which requires a paradigm shift from the assumption of $100 \%$ reliable computations to fault-tolerant signal-processing systems. In this paper, we illustrate how the impact of unreliable circuits can be taken into account in the performance analysis of wireless communication systems. To this end, we consider the example of a multiple-input multiple-output (MIMO) system using bit-interleaved coded modulation in which a soft-output detector delivers quantized log-likelihood ratios (LLRs) to the channel decoder through an interleaver memory suffering from bit-flips due to reliability issues of the silicon implementation.
\end{abstract}

\section{INTRODUCTION}

One of the key advantages of digital signal-processing (DSP) systems has always been the ability to store data and carry out computations in a well-defined and $100 \%$ reproducable manner. Unfortunately, it is now becoming more and more important to realize that very large scale integrated (VLSI) circuits manufactured in advanced silicon process technologies can no longer live up to these expectations. Unreliable behavior of integrated circuits is already observed today for example in space applications, where ionizing radiation can randomly alternate the content of on-chip storage devices. In the near future, similar issues are expected to appear more often, even in consumer electronics devices. In particular, the transition to deep-submicron integrated circuits having feature sizes below $32 \mathrm{~nm}$ and the strive for ultra low power consumption [1] will no longer allow us to naively assume that computations are always carried out correctly and that data can be stored reliably in high-density on-chip memories. Therefore, realizing DSP circuits in future silicon technologies will require a paradigm shift from the today's assumption of $100 \%$ reliable operation to fault-tolerant signal processing systems [2], [3]. Such systems must take the presence of errors in the data induced by the circuit implementation into account and must be robust against such errors.

Wireless communication receivers naturally deal with signals that are already corrupted by noise and interference. Resilience against this type of distortion is typically achieved by the use of channel coding to ensure reliable data transmission. This inherent fault-tolerance of wireless transceivers and the availability of information-theoretic performance metrics provides the motivation to study the impact of unreliable circuits on the performance of communication systems and to investigate options to provide robustness against circuit failures ${ }^{1}$.

\section{A. Case-Study: MIMO-BICM System}

In this paper, we consider the example of a multiple-input multiple-output (MIMO) system with bit-interleaved coded modulation (BICM) in which a block of information bits is mapped to transmit symbols via a channel encoder and a symbol mapper separated by a code-bit interleaver [5], [6]. At the receiver side, a detector calculates log-likelihood ratios (LLRs) for the coded bits, which are deinterleaved and passed to the subsequent channel decoder. Theoretically, one realvalued LLR value per code bit needs to be computed and stored in the receiver. Clearly, digital receiver implementations can only use finite word-length representation of real numbers, which motivates the study of LLR quantization. Optimal LLR quantization that maximizes the information rate for the special case of BPSK modulation over an AWGN channel was considered previously in [7], while [8] considered LLR quantization for MIMO-BICM systems with more general channels and higher-order symbol alphabets. The purpose of these optimizations is to reduce the amount of storage required for the deinterleaver since it accounts for a considerable portion of the overall receiver silicon area and power consumption [9].

\section{B. Contributions}

Arguing that this significant amount of storage is a part of the silicon realization that is particularly sensitive to circuit failures we extend the performance analysis and optimization in [8] to the case where quantized LLRs are stored in unreliable on-chip memories.

To this end, we study the consequences on the performance of the overall communication system using informationtheoretic measures to characterize the performance and to devise methods to make the system more robust against errors

\footnotetext{
${ }^{1}$ We further note that the considered topic is also relevant in the case of a network-coding or relaying scenario, where nodes in a wireless network exchange information between cooperating nodes through unreliable (e.g. wireless) communication channels [4].
} 


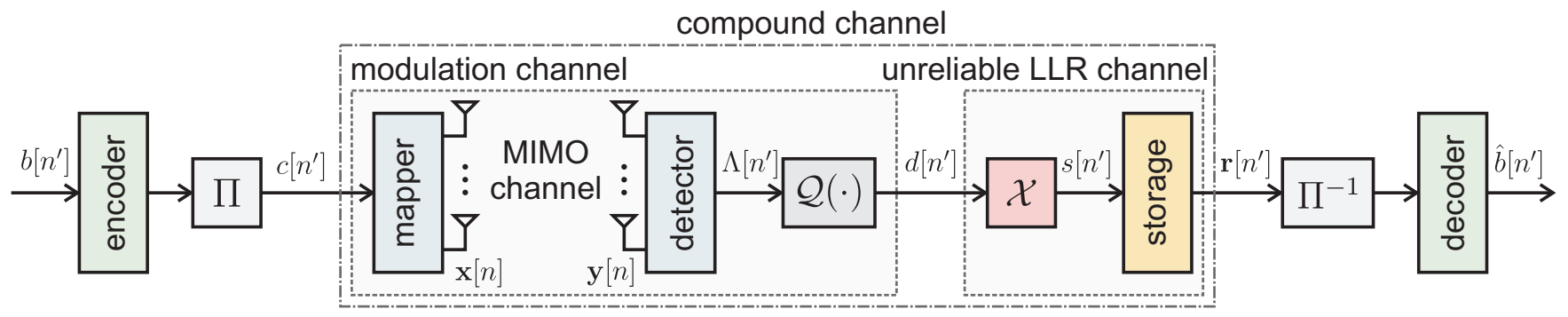

Fig. 1. Block diagram of a MIMO-BICM system with LLR quantization and an unreliable LLR channel.

occurring in the storage of LLRs. More specifically, our contributions are as follows:

- We model the unreliable LLR storage or transmission as a binary symmetric channel (BSC), introducing i.i.d. bit flips on the LLR's bit labels.

- We investigate the performance loss caused by the unreliable LLR channel. To this end, we characterize the performance through the mutual information of the overall equivalent channel [10].

- We compare the effect of different binary number representations on the system performance. We also allow for some redundancy in the labeling and study the optimal labeling strategy. Furthermore, we consider optimum LLR quantization that maximizes the overall performance for a fixed labeling.

- For larger word-lengths, finding the optimal labeling becomes computationally infeasible. We therefore propose to encode the bit labels by means of an error-correcting code. We explore how the word-lengths should be allocated between LLR quantization and error correction and give related design guidelines.

Outline: The remainder of this paper is organized as follows. Sec. II introduces the MIMO-BICM system model and details the model for unreliable LLR storage. In Sec. III we provide simulation results and discuss the impact of unreliable LLR storage to the mutual information of the overall communication system. We conclude in Sec. IV.

\section{System Model}

We consider a MIMO-BICM system with $M_{T}$ transmit antennas and $M_{R}$ receive antennas; a corresponding block diagram is depicted in Fig. 1. The system includes the softoutput detector delivering quantized LLRs, which are passed through the unreliable LLR channel and the channel decoder.

\section{A. Transmission Model}

A sequence of information bits $b\left[n^{\prime}\right]$ is encoded using an error-correcting code and passed through a bitwise interleaver $\Pi$ generating a pseudo-random sequence $c\left[n^{\prime}\right]$. The uniformly distributed and interleaved code bits are demultiplexed into $M_{T}$ antenna streams ("layers"). In each layer, groups of $m$ code bits are mapped to (complex) data symbols $x_{k}[n] \in \mathcal{A}$, $k=1, \ldots, M_{T}$; here, $\mathcal{A}$ denotes the symbol alphabet of size $|\mathcal{A}|=2^{m}$. The transmit vector at symbol time $n$ is given by $\mathbf{x}[n] \triangleq\left(x_{1}[n] \cdots x_{M_{T}}[n]\right)^{T}$ and carries $R_{0} \triangleq m M_{T}$ interleaved code bits $c_{\ell}[n], \ell=1, \ldots, R_{0}$.

Assuming flat fading, the length- $M_{R}$ receive vector results from the following input-output relation:

$$
\mathbf{y}[n]=\mathbf{H}[n] \mathbf{x}[n]+\mathbf{w}[n] .
$$

Here, $\mathbf{H}[n]$ designates the complex-valued $M_{R} \times M_{T}$ MIMO channel matrix and $\mathbf{w}[n] \sim \mathcal{C N}\left(\mathbf{0}, \sigma^{2} \mathbf{I}\right)$ denotes the complexvalued Gaussian noise vector. In the following, we will omit the symbol-time index $n$ in order to simplify notation.

At the receiver, a max-log detector computes LLRs for each code bit $c_{\ell}$ according to [11], [12]

$$
\Lambda_{\ell}=\frac{1}{\sigma^{2}}\left[\min _{\mathbf{x} \in \mathcal{X}_{\ell}^{0}}\|\mathbf{y}-\mathbf{H} \mathbf{x}\|^{2}-\min _{\mathbf{x} \in \mathcal{X}_{\ell}^{1}}\|\mathbf{y}-\mathbf{H} \mathbf{x}\|^{2}\right]
$$

where $\mathcal{X}_{\ell}^{b}$ denotes the set of transmit vectors for which $c_{\ell}=b$.

\section{B. LLR Quantization}

We consider a $q$-bit quantizer characterized by $K=2^{q}$ bins $\mathcal{I}_{k}=\left[i_{k-1}, i_{k}\right], k=1, \ldots, K$. We use the convention $i_{0}=-\infty, i_{K}=\infty$, with boundaries $i_{k}$ sorted in ascending order. The quantizer $\mathcal{Q}(\cdot)$ maps the LLR $\Lambda_{\ell}$ to a discrete LLR $d_{\ell}$ according to

$$
d_{\ell}=\mathcal{Q}\left(\Lambda_{\ell}\right)=\lambda_{k} \quad \text { if } \Lambda_{\ell} \in \mathcal{I}_{k},
$$

where $\lambda_{k} \in \mathcal{I}_{k}$ stands for the $k$ th quantization level. In the following, we consider the equivalent discrete channel with binary-valued input $c \in\{0,1\}$ and $K$-ary output $d \in\left\{\lambda_{1}, \ldots, \lambda_{K}\right\}$. Here, $c$ and $d$ are obtained by randomly picking a bit position $\ell=1, \ldots, R_{0}$ according to a uniform distribution. This models a situation where the outer channel code is "blind" to the bit positions within the symbol labels. The equivalent channel is characterized by the crossover probabilities

$$
p_{b k}=\operatorname{Pr}\left\{d=\lambda_{k} \mid c=b\right\}=\operatorname{Pr}\left\{\Lambda \in \mathcal{I}_{k} \mid c=b\right\}
$$

which can be obtained according to

$$
p_{b k}=\int_{\mathcal{I}_{k}} f_{\Lambda \mid c}(\xi \mid b) d \xi
$$

with $f_{\Lambda \mid c}(\xi \mid b)$ denoting the conditional probability density function (pdf) of the LLR $\Lambda$ given that $c=b$. The mutual 


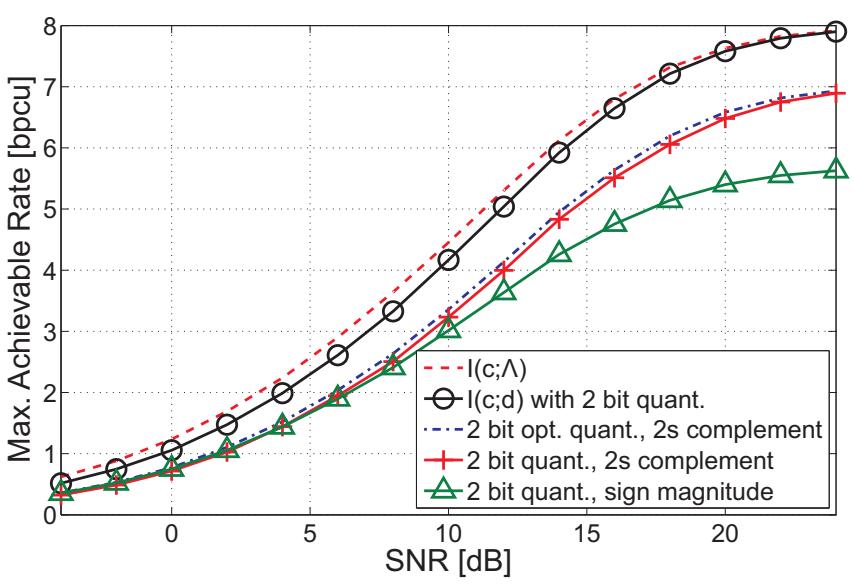

(a) Comparison of $2 \mathrm{~s}$ complement and sign-magnitude number representations with quantization to 2 bit.

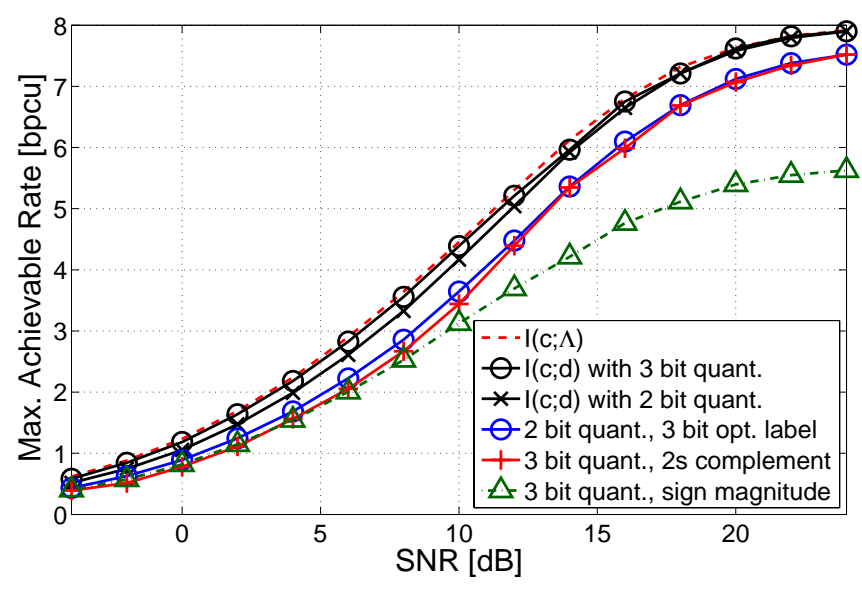

(b) Comparison of different number representations and labeling strategies for 2 bit and 3 bit LLR quantization.

Fig. 2. Maximum achievable rates in a $M_{R}=M_{T}=2$ MIMO-BICM system using a Gray-mapped 16-QAM symbol alphabet.

information $I(c ; d)$ [13] between coded bits $c$ and quantizer output $d$ is given by

$$
I(c ; d)=\frac{1}{2} \sum_{b=0}^{1} \sum_{k=1}^{K} p_{b k} \log _{2} \frac{2 p_{b k}}{p_{0 k}+p_{1 k}} .
$$

In this work, the LLR distribution $f_{\Lambda \mid c}(\xi \mid b)$ and hence the transition probabilities $p_{b k}$ are averaged with respect to the statistics of the physical channel $\mathbf{H}$ (reflecting fast fading); therefore the quantity $I$ describes the ergodic rate achievable over the equivalent channel (cf. [14]).

In the following, we will consider two different quantizer designs. The first is a uniform quantizer, which quantizes the LLRs $\Lambda_{\ell}$ into $K$ bins in a uniform manner. Second, we consider the information-theoretic optimal quantizer [8]; here, the quantizer interval boundaries $i_{k}$ are chosen such that the mutual information $I(c ; d)$ of the equivalent channel [5] is maximized. In general, no closed-form expression for the conditional densities $f(\Lambda \mid c)$ are available and the mutual information depends on the quantizer intervals $i_{k}$ in a complicated way. Therefore, the optimal quantizer is designed numerically based on Monte-Carlo simulations.

\section{Unreliable LLR Storage}

Before the quantized LLRs delivered by the detector are decoded, they are reordered in the deinterleaver. This deinterleaver is essentially comprised of a large on-chip memory that is assumed to be the source of errors due to unreliable silicon implementations. In our model, we separate this storage from the interleaver by introducing an additional unreliable memory before the assumed reliable interleaver operation (cf., Fig. 1). To store quantized LLRs $d_{\ell}$ in this memory, they are assigned binary-valued labels of length $L$. We allow for a larger number of different labels than quantization intervals, i.e., $q \leq L$, so that we are able to add some redundancy. The $k$ th quantizer output is assigned a binary-valued label denoted by $\mathbf{s}_{k}$ by means of the mapper $\chi$. After storage in the unreliable memory, the bit label $\mathbf{r}$ is observed. We model the unreliable LLR storage as a binary symmetric channel (BSC), introducing i.i.d. bit flips on the LLR's bit labels with crossover probability $\epsilon$. The conditional probability of observing the label $\mathbf{r}_{n}$, having transmitted the label $\mathbf{s}_{k}$, therefore becomes

$$
\operatorname{Pr}\left(\mathbf{r}=\mathbf{r}_{n} \mid \mathbf{s}=\mathbf{s}_{k}\right)=\epsilon^{d_{H}\left(\mathbf{r}_{n}, \mathbf{s}_{k}\right)}(1-\epsilon)^{L-d_{H}\left(\mathbf{r}_{n}, \mathbf{s}_{k}\right)} .
$$

Here, $d_{H}\left(\mathbf{r}_{n}, \mathbf{s}_{k}\right)$ denotes the Hamming distance between the binary-valued labels $\mathbf{r}_{n}$ and $\mathbf{s}_{k}$.

The combination of LLR quantization and LLR transmission yields a compound channel (see Fig. 1) with binaryvalued input $c$ and output $\mathbf{r}$. The conditional probability $\operatorname{Pr}\left(\mathbf{r}=\mathbf{r}_{n} \mid b\right)$ of this compound channel is given by

$$
\bar{p}_{b n} \triangleq \operatorname{Pr}\left(\mathbf{r}=\mathbf{r}_{n} \mid b\right)=\sum_{k=1}^{2^{L}} \operatorname{Pr}\left(\mathbf{r}=\mathbf{r}_{n} \mid \mathbf{s}=\mathbf{s}_{k}\right) p_{b k} .
$$

Based on these conditional probabilities, the mutual information $I(c ; \mathbf{r})$ of the compound channel is obtained as in (4) with $p_{b k}$ replaced by $\bar{p}_{b n}$, i.e.,

$$
I(c ; \mathbf{r})=\frac{1}{2} \sum_{b=0}^{1} \sum_{n=1}^{2^{L}} \bar{p}_{b n} \log _{2} \frac{2 \bar{p}_{b n}}{\bar{p}_{0 n}+\bar{p}_{1 n}} .
$$

After receiving the bit label $\mathbf{r}$, the LLR values need to be reconstructed prior to be fed into the channel decoder, which finally yields hard-decisions on the information bits. The reconstructed LLRs provide the channel decoder with accurate reliability information, which is given by the crossover probabilities of the compound channel. We therefore propose to use LLRs which are obtained according to [15]

$$
\bar{\Lambda}=\log \frac{\bar{p}_{1 n}}{\bar{p}_{0 n}} .
$$

The channel decoder employs, for example, a Viterbi decoder or a belief-propagation algorithm which yields the decisions $\hat{b}$ for the information bits. 


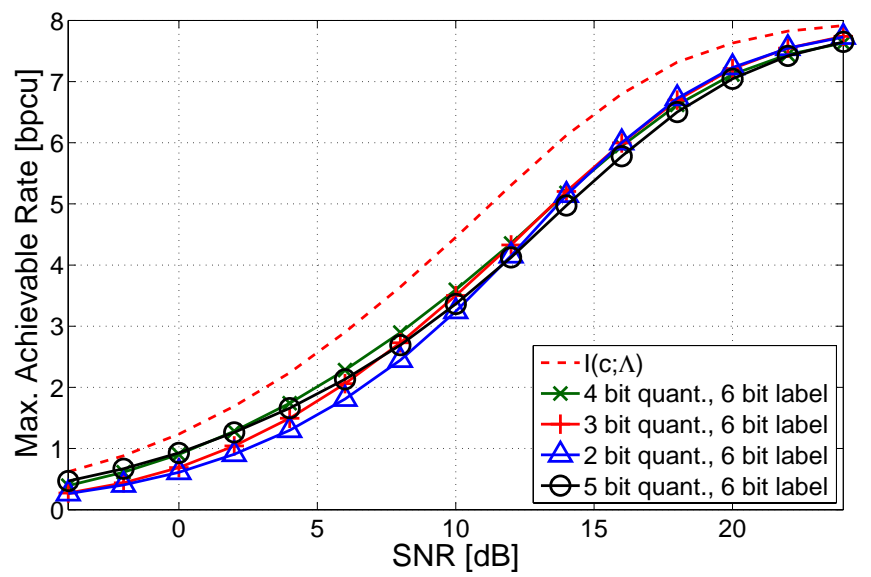

(a) Binary-valued labels of length $L=6$ bit.

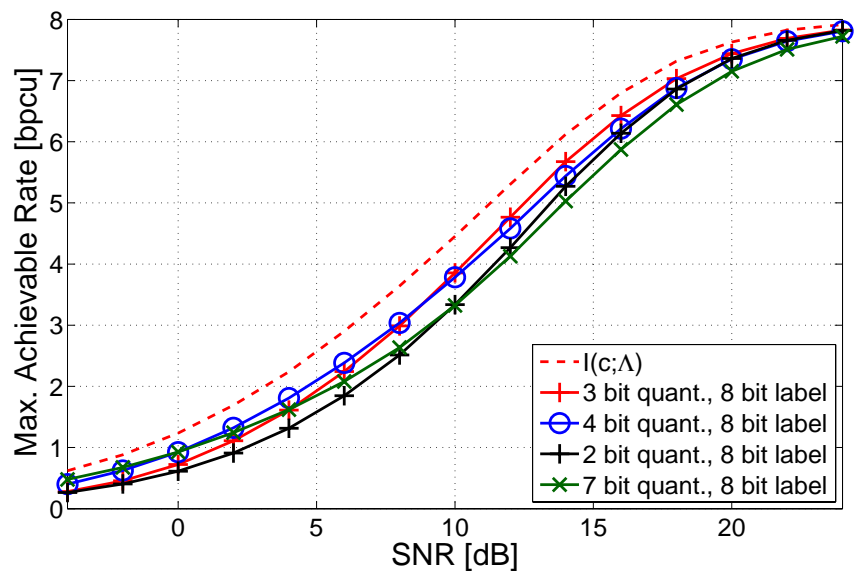

(b) Binary-valued labels of length $L=8$ bit.

Fig. 3. Maximum achievable rate for a $M_{R}=M_{T}=2$ MIMO-BICM system using 16-QAM. The binary-valued labels are encoded by binary cyclic code and using different quantization word-lengths.

\section{Simulation Results AND Discussion}

In the following, we present simulation results for a $M_{R}=$ $M_{T}=2$ MIMO-BICM system with a Gray mapped 16QAM symbol alphabet. The MIMO channel is assumed to be i.i.d. Rayleigh fading, and the bit-flip probability of the LLR channel corresponds to $\epsilon=0.05$.

\section{A. Optimal Quantizer}

Fig. 2(a) shows achievable rates of different number representations using the 2 bit (information theoretic) optimal quantizer. As reference, we show the achievable rate $I(c ; d)$ for error-free memories using the information theoretic optimal quantizer with 2 bit quantization and the maximum achievable rate $I(c ; \Lambda)$ without LLR quantization. For a wide range of rates, the 2 bit quantization suffers an SNR-performance loss of roughly $1 \mathrm{~dB}$.

The curves associated with " 2 bit quant., 2 s complement" and " 2 bit quant., sign magnitude" show the achievable rates $I(c ; \mathbf{r})$ of the compound channel, using 2 s complement and sign magnitude [16] for representing the 2 bit labels, respectively. The 2 s complement number representation clearly outperforms the sign magnitude representation; at a rate of 4 bpcu the SNR gap is about $2.5 \mathrm{~dB}$. This can be explained as follows: Using sign-magnitude representation, the bit-flip of the sign bit causes a large error in the reconstructed LLR $\bar{\Lambda}$, which reduces the achievable rate. We can clearly observe that the number representation (or more generally the labeling) has a significant impact on the overall system performance and therefore, needs to be chosen carefully.

Being interested in the performance of the compound system, we propose to design the quantizer such that the mutual information $I(c ; \mathbf{r})$ of the compound channel is maximized. Numerically optimizing the quantizer for the 2 s complement number representation yields the curve labeled "2 bit opt. quant., 2s complement" in Fig. 2(a). It shows only a slight improvement compared to the achievable rate of the system employing a quantizer maximizing $I(c ; d)$.

We next fix the length of the binary-valued label to $L=3$ bit and compare the impact of different quantizer word-length and number representations in Fig. 2(b). As a reference, we plot the achievable rate $I(c ; \Lambda)$, and $I(c ; d)$ with 3 bit and 2 bit (information-theoretic optimal) quantization, respectively. Similar to the 2 bit case, 3 bit quantization using signmagnitude labeling performs worse.

The curve labeled " 2 bit quant., 3 bit opt. label" refers to the case, where the LLRs are quantized with 2 bit, but 3-bit labels are used. The bit labeling is optimized by a brute-force search over all $2^{3} ! / 2^{2}$ ! possible mappings. For rates below $6 \mathrm{bpcu}$, this approach can be seen to outperform 3 bit quantization with $2 \mathrm{~s}$ complement representation. From this result we can draw the conclusion, that it might be better to quantize with fewer bits and allow for some redundancy in the bit labels, instead of using all available bits only for quantization.

We finally note that $I(c ; d) \rightarrow 8$ bpcu in the limit of high SNR, while $I(c ; \mathbf{r})$ does not converge to this limit. This different behavior is due to the (SNR independent) performance loss caused by the unreliable LLR transmission. Hence, in the high-SNR regime, the dominating errors will be caused by the "unreliable LLR channel" rather than the wireless ("modulation") channel, which eventually requires to protect the stored bits against corruptions (e.g., through coding).

\section{B. Uniform Quantizer with an Error-Correcting Code}

For larger values of the label-width $L$, it is computationally infeasible to search for the optimal labeling. Instead, we propose to encode the quantizer outputs by means of a cyclic $(L, K)$ binary block code in order to add redundancy. Due to its simple design, we employ a uniform LLR quantizer. We consider quantization with different word-lengths $K$, and fix the total bit length to $L=6$ bit and $L=8$ bit, respectively. The obtained achievable rates are shown in Fig. 3(a) and (b), 


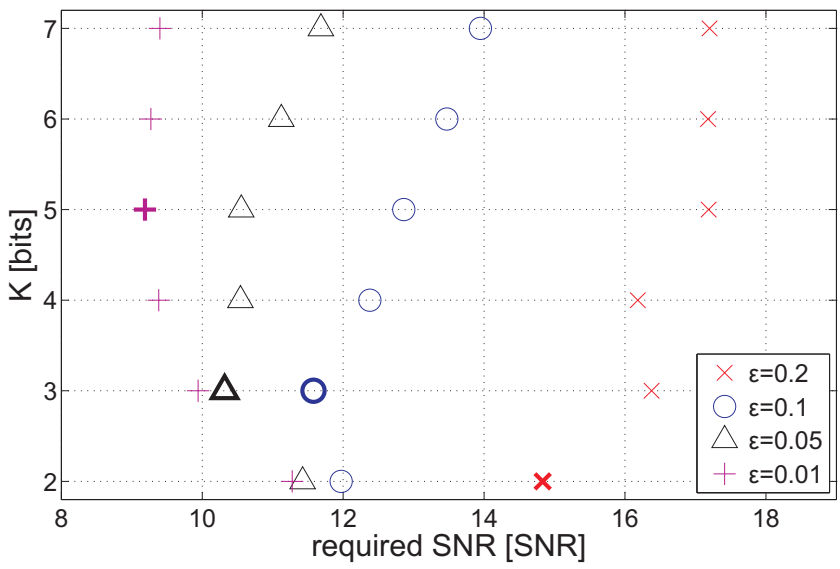

Fig. 4. Required SNR for a target rate of $R=4 \mathrm{bpcu}$ versus quantization word-length $K$ for different values of bit-flip probability $\epsilon$.

respectively. As reference, we also show the achievable rate $I(c ; \Lambda)$.

At rates of $R=4 \mathrm{bpcu}$, it seems to be optimal in both cases to use 3 bit or 4 bit for quantization. The resulting SNRperformance gaps to the unquantized case are $2 \mathrm{~dB}$ and $1.5 \mathrm{~dB}$, respectively. Quantization using more bits leads to a higher mutual information $I(c ; d)$, but reduces the redundancy that can be exploited to provide robustness against implementation failures and therefore incurs a performance loss. Using less bits for quantization allows for better error protection of the bit labels, but incurs a higher quantization loss.

In case of $L=6$, larger word-lengths for the quantizer perform better in case of low rates, while 2 bit or 3 bit quantization is optimal for high rates. When using labeling of word-lengths $L=8$ bit, 4 bit quantization is optimal at low rates, while 3 bit quantization performs best for high rates.

\section{Impact of the Bit-Flip Probability}

We finally study the effect of the bit-flip probability on the performance of the system. In Fig. 4 we plot the required SNR for a target rate of $R=4$ bpcu versus quantization wordlengths $K$ for different values of crossover probabilities $\epsilon$. It can be observed that for low values of bit-flip probability $\epsilon$ quantization with $K=4$ bit is optimal, whereas in case of high $\epsilon$ quantization with only $K=2$ bit is optimal. This clearly demonstrates that highly unreliable LLR transmission channels require better error-protection (and thus smaller quantization word-lengths) in order to achieve the best performance.

\section{CONCLUSiON}

Future signal-processing systems need to be robust against unreliable circuit implementations that can no longer guarantee $100 \%$ reliable operation. In this paper we have studied the impact of unreliable LLR storage on the performance of a MIMO-BICM wireless communication system. To this end, we have characterized the system performance based on the mutual information of the channel, the LLR quantizer, and a simple unreliable-storage circuit-model used for the deinterleaver. We observed that reasonable performance can be maintained even at bit-error rates in the storage device of $5 \%$. If no additional bits are used for the LLR representation compared to an error-free baseline implementation, we find that the choice of a suitable mapping of LLRs to binary-labels is crucial to minimize the performance loss. If additional bits are allowed to protect against errors in the silicon implementation, we find that in the low-SNR regime, these bits should be used primarily to provide better number representation of quantized LLRs. On the other hand, in the high-SNR regime, the additional bits should be used primarily for redundancy through coding to protect against circuit errors.

\section{ACKNOWLEDGMENTS}

This work was funded by the SNSF under the project number PP002-119052, the FWF Grant N10606, and the STREP project MASCOT (IST-026905) within the Sixth Framework Programme of the European Commission. The authors wish also to acknowledge the activity of the Network of Excellence in Wireless COMmunications NEWCOM++ of the European Commission (contract no. 216715).

\section{REFERENCES}

[1] Z. Bo, S. Hanson, D. Blaauw, and D. Sylvester, "A variation-tolerant sub$200 \mathrm{mV}$ 6-T subthreshold SRAM,” IEEE Journal of Solid-State Circuits, vol. 43, pp. 2338-2348, Oct. 2008

[2] M. A. Breuer, "Let's think analog," in Proc. of the IEEE Computer Society Annual Symposium on VLSI, pp. 2-5, May 2005.

[3] S. Ghosh and K. Roy, "Parameter variation tolerance and error resiliency: New design paradigm for the nanoscale era," Proceedings of the IEEE, vol. 98, pp. $1718-1751$, Oct. 2010.

[4] S. Schwandter and G. Matz, "A practical forwarding scheme for wireless relay channels based on the quantization of log-likelihood ratios," in Proc. IEEE ICASSP, (Dallas, Texas), pp. 2502-2505, March 2010.

[5] A. G. i Fàbregas, A. Martinez, and G. Caire, "Bit-interleaved coded modulation," Foundations and Trends in Communications and Information Theory, vol. 5, no. 1-2, pp. 1-153, 2008.

[6] G. Caire, G. Taricco, and E. Biglieri, "Bit-interleaved coded modulation,” IEEE Trans. Inf. Theory, vol. 44, pp. 927-945, May 1998.

[7] W. Rave, "Quantization of log-likelihood ratios to maximize mutual information," IEEE Signal Processing Letters, vol. 16, pp. 283-286, Apr. 2009.

[8] C. Novak, P. Fertl, and G. Matz, "Quantization for soft-output demodulators in bit-interleaved coded modulation systems," in Proc. IEEE ISIT2009, (Seoul, Korea), pp. 1070-1074, 2009.

[9] C. Roth, P. Meinerzhagen, C. Studer, and A. Burg, "A 15.8 pJ/bit/iter quasi-cyclic LDPC decoder for IEEE 802.11n in $90 \mathrm{~nm}$ CMOS," in Proc. IEEE Asian Solid-State Circuits Conf., Nov. 2010.

[10] J. Jaldén, P. Fertl, and G. Matz, "On the generalized mutual information of BICM Systems with approximate demodulation," in Proc. IEEE ITW 2010, (Cairo, Egypt), Jan. 2010.

[11] S. H. Müller-Weinfurtner, "Coding approaches for multiple antenna transmission in fast fading and OFDM," IEEE Trans. Signal Processing, vol. 50, pp. 2442-2450, Oct. 2002.

[12] C. Studer, A. Burg, and H. Bölcskei, "Soft-output sphere decoding: Algorithms and VLSI implementation," IEEE J. Sel. Areas Comm., vol. 26, pp. 290-300, Feb. 2008.

[13] T. M. Cover and J. A. Thomas, Elements of Information Theory. New York: Wiley, 1991.

[14] D. Tse and P. Viswanath, Fundamentals of Wireless Communication. Boston (MA): Cambridge University Press, 2005.

[15] P. Fertl, J. Jaldén, and G. Matz, "Capacity-based performance comparison of MIMO-BICM demodulators," in Proc. IEEE SPAWC-08, (Recife, Brazil), pp. 166-170, July 2008.

[16] H. Kaeslin, Digital integrated circuit design: from VLSI architectures to CMOS fabrication. Cambridge, UK: Cambridge University Press, 2008 